\title{
Asiatic acid attenuates hypertrophic and fibrotic differentiation of articular chondrocytes via AMPK/PI3K/AKT signaling pathway
}

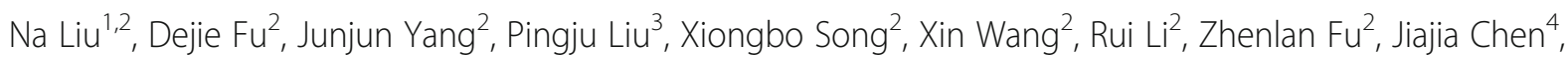
Xiaoyuan Gong ${ }^{2}$, Cheng Chen ${ }^{5^{*}}$ (i) and Liu Yang ${ }^{2^{*}}$

\begin{abstract}
Background: Osteoarthritis $(\mathrm{OA})$, the most common joint disorder, is characterized by a progressive degradation of articular cartilage. Increasing evidence suggests that $\mathrm{OA}$ is closely associated with cartilage pathologies including chondrocyte hypertrophy and fibrosis.

Methods: In this study, we showed that asiatic acid (AA) treatment reduced chondrocyte hypertrophy and fibrosis. First, the cytotoxicity of $\mathrm{AA}(0,5,10$, and $20 \mu \mathrm{M})$ to chondrocytes was evaluated, and $5 \mu \mathrm{M}$ was selected for subsequent experiments. Then, we detected the gene and protein level of chondrocyte hypertrophic markers including type $X$ collagen (COL-X), matrix metalloproteinase-13 (MMP-13), alkaline phosphatase (ALP), and runtrelated transcription factor 2 (Runx2); chondrocyte fibrosis markers including type I collagen (COL-I) and alphasmooth muscle actin (a-SMA); and chondrogenic markers including SRY-related HMG box 9 (SOX9), type II collagen (COL-II), and aggrecan (ACAN). Further, we tested the mechanism of AA on inhibiting chondrocyte hypertrophy and fibrosis. Finally, we verified the results in an anterior cruciate ligament transection (ACLT) rat OA model.

Results: We found that AA treatment inhibited the hypertrophic and fibrotic phenotype of chondrocytes, without affecting the chondrogenic phenotype. Moreover, we found that AA treatment activated AMP-activated protein kinase (AMPK) and inhibited phosphoinositide-3 kinase/protein kinase B (PI3K/AKT) signaling pathway in vitro. The results in an ACLT rat OA model also indicated that AA significantly attenuated chondrocyte hypertrophy and fibrosis.

Conclusion: AA treatment could reduce hypertrophic and fibrotic differentiation and maintain the chondrogenic phenotype of articular chondrocytes by targeting the AMPK/PI3K/AKT signaling pathway. Our study suggested that AA might be a prospective drug component that targets hypertrophic and fibrotic chondrocytes for OA treatment.
\end{abstract}

Keywords: Asiatic acid, Chondrocytes, Osteoarthritis, Hypertrophy, Fibrosis, AMPK, PI3K, AKT

\footnotetext{
* Correspondence: ccllijf@163.com; jointsurgery@163.com

${ }^{5}$ College of Medical Informatics, Chongqing Medical University, Chongqing,

China

${ }^{2}$ Center for Joint Surgery, Southwest Hospital, Third Military Medical

University (Army Medical University), Chongqing, China

Full list of author information is available at the end of the article
}

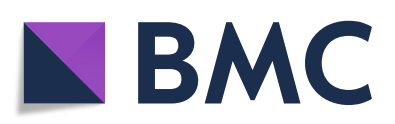

(- The Author(s). 2020 Open Access This article is licensed under a Creative Commons Attribution 4.0 International License, which permits use, sharing, adaptation, distribution and reproduction in any medium or format, as long as you give appropriate credit to the original author(s) and the source, provide a link to the Creative Commons licence, and indicate if changes were made. The images or other third party material in this article are included in the article's Creative Commons licence, unless indicated otherwise in a credit line to the material. If material is not included in the article's Creative Commons licence and your intended use is not permitted by statutory regulation or exceeds the permitted use, you will need to obtain permission directly from the copyright holder. To view a copy of this licence, visit http://creativecommons.org/licenses/by/4.0/. The Creative Commons Public Domain Dedication waiver (http://creativecommons.org/publicdomain/zero/1.0/) applies to the data made available in this article, unless otherwise stated in a credit line to the data. 


\section{Introduction}

Chondrocyte hypertrophy plays an essential role in endochondral ossification during bone formation and growth [1]. However, ectopic hypertrophy of articular chondrocytes has been recognized as an osteoarthritis (OA)-promoting factor [2-4]. In early stages of OA, articular chondrocytes respond to the degenerative events by decreasing the production of type II collagen (COL-II) and increasing the synthesis of type $\mathrm{X}$ collagen (COL-X), which is an indication of chondrocyte hypertrophy [5]. Meanwhile, activity of matrix metalloproteinase-13 (MMP-13), a major collagenase expressed in hypertrophic chondrocytes, has been observed with OA onset [6]. In addition, a significantly increased expression of alkaline phosphatase (ALP) and runt-related transcription factor 2 (Runx2) is identified in osteoarthritic chondrocytes [7, 8]. Kamekura et al. investigated the functional involvement of Runx2 in chondrocyte hypertrophy and OA development and found that compared with wildtype mice, Runx2-deficient mice had no hypertrophic chondrocytes and showed a decrease in cartilage destruction, along with a reduction in COL-X and MMP-13 expression [9]. Therefore, chondrocyte hypertrophy is closely related to OA pathogenesis.

On the other hand, dedifferentiation of articular chondrocytes usually occurs during in vitro culture and expansion [10], which is characterized by the loss of COLII and increase in type I collagen (COL-I) $[11,12]$. The phenotypic instability and easy access to the fibroblastlike phenotype seriously affect the outcome of cartilage repair when using chondrocytes as seed cells. Taken together, how to inhibit chondrocyte hypertrophy and chondrocyte dedifferentiation is of great concern in both OA treatment and cartilage tissue engineering.

Asiatic acid (AA), a pentacyclic triterpene isolated from Centella asiatica [13], has been reported to exhibit a variety of pharmacological effects, including antioxidant, anti-inflammatory, and hepatoprotective activities [14-16]. Particularly, recent studies demonstrate that AA inhibits cardiac hypertrophy [17] and liver fibrosis [18]. However, whether AA could attenuate the hypertrophic differentiation or the fibrotic differentiation of articular chondrocytes has not been reported. We hypothesized that AA might attenuate chondrocyte hypertrophy or chondrocyte dedifferentiation. To verify this hypothesis, first we treated human osteoarthritic chondrocytes with AA and measured the changes of hypertrophic markers and fibrotic markers; then, we intraarticularly injected AA in a rat OA model and analyzed the joint histology after 4 weeks and 8 weeks.

\section{Materials and methods}

\section{Chemicals}

Asiatic acid (purity >97.0\%; molecular weight 488.70), purchased from Sigma-Aldrich (St. Louis, USA), was dissolved in dimethylsulfoxide (DMSO) as a 2-mM stock solution and stored at $4{ }^{\circ} \mathrm{C}$. Further dilution was done in cell culture medium.

\section{Cell isolation and culture}

Cartilage samples were obtained intraoperatively from patients $(n=3$, age $55 \pm 10)$ undergoing total knee arthroplasties with approval from the Ethics Committee of Southwest Hospital (Chongqing, China). Chondrocytes were isolated according to our previous protocol [19]. Briefly, cartilage pieces were digested in highglucose DMEM (C11995500BT, Gibco, USA) supplemented with $0.2 \%$ type II collagenase (C6885, Sigma, USA) and $1 \%$ penicillin/streptomycin $(\mathrm{P} / \mathrm{S})$ overnight at $37^{\circ} \mathrm{C}$. The resulting cell suspension was filtered through a $40-\mu \mathrm{m}$ cell strainer; collected cells were centrifuged ( $400 \mathrm{~g}$ for $5 \mathrm{~min}$ ) and resuspended in high-glucose DMEM supplemented with $10 \%$ fetal bovine serum (FBS; Hyclone, USA) and $1 \% \mathrm{P} / \mathrm{S}$. Finally, cells were plated at a density of $1 \times 10^{5}$ cells per well in 6-well plates and incubated in a humidified atmosphere of $5 \%$ $\mathrm{CO}_{2}$ at $37^{\circ} \mathrm{C}$. The medium was changed every 2-3 days. Only cells at passage 1 were used in our study to avoid phenotype loss.

\section{Live-dead cell staining and cell viability assay}

The effects of AA on the viability of chondrocytes were evaluated using a Live/Dead staining kit (40747ES76, Yeasen, China). Briefly, after $24 \mathrm{~h}$ treatment of AA (0, 5, 10, and $20 \mu \mathrm{M})$, chondrocytes were incubated with $2 \mu \mathrm{M}$ Calcein-AM and $4.5 \mu \mathrm{M}$ PI for $15 \mathrm{~min}$ at room temperature (RT) in the dark. Labeled cells were visualized using a confocal microscope (IX71, Olympus, Japan). Live cells were stained green, whereas dead cells were stained red.

To further evaluate the cytotoxicity of AA, measurement of cell viability was performed using the Cell Counting Kit-8 (CCK-8; CK04, Do Jindo Laboratories, Japan). Chondrocytes were cultured in 96-well plates at a density of $5 \times 10^{3}$ cells per well for $24 \mathrm{~h}$. Then, cells were pretreated with $\mathrm{AA}$ at different concentrations $(0,5,10$, and $20 \mu \mathrm{M})$ for $24 \mathrm{~h}$. After that, $10 \mu \mathrm{L}$ CCK-8 solution was added to each well and incubated at $37^{\circ} \mathrm{C}$ for $2 \mathrm{~h}$. The optical density was read at a wavelength of $450 \mathrm{~nm}$ with a microplate reader (Thermo Fisher Scientific, USA).

\section{Alcian Blue staining}

The cells were washed with PBS and fixed with $4 \%$ formaldehyde for $10 \mathrm{~min}$ at RT. Then, the cells were washed three times with PBS and stained with Alcian Blue (Cyagen, USA) for $30 \mathrm{~min}$. The cells were washed again three times with PBS and imaged. 


\section{Alkaline phosphatase staining}

Cells were cultured in 24-well plates at a density of $1 \times$ $10^{4}$ cells per well, followed by stimulation with AA. After 3 days of culturing, the cells were washed with PBS and stained using an ALP staining kit (C3206, Beyotime, China) according to the manufacturer's protocol. The cells were washed again three times with PBS and imaged.

\section{Immunofluorescence staining}

Cells were washed with PBS and fixed with $4 \%$ formaldehyde for $10 \mathrm{~min}$ at RT. Then, cells were washed three times with cold PBS and treated with Triton X-100 (P0096, Beyotime) for $10 \mathrm{~min}$ at RT. Cells were washed again three times with PBS and blocked $1 \mathrm{~h}$ with Blocking Buffer (P0260, Beyotime) at RT followed by incubation with the primary antibodies: COL-II (dilution of 1 : 200; ab34712, Abcam, USA), Aggrecan (dilution of 1: 100; ab3778, Abcam), SOX9 (dilution of 1:250; ab185230, Abcam), MMP-13 (dilution of 1:200; ab39012, Abcam), COL-X (dilution of 1:50; ab58632, Abcam), Runx2 (dilution of 1:500; ab23981, Abcam), COL-I (dilution of 1:200; ab34710, Abcam), and $\alpha$-SMA (dilution of 1:500; A5228, Sigma) overnight at $4{ }^{\circ} \mathrm{C}$. Next, the cells were washed three times with PBST, incubated with secondary antibody for $1 \mathrm{~h}$ at RT. Cell nuclei were counterstained with DAPI for $5 \mathrm{~min}$ at $37^{\circ} \mathrm{C}$, and the images were obtained by confocal fluorescent microscope (LSM710, Carl Zeiss, Germany).

\section{Reverse transcription-polymerase chain reaction}

RT-PCR total RNA was isolated from chondrocytes using RNA pure Total RNA Kit9 (RP5612, BioTeke, China) following the manufacturer's protocol. RNA concentration was determined spectrophotometrically using a NanoDrop ND1000 spectrophotometer (Isogen Life Science B.V., Netherlands). The A260/A280 ratio was calculated to verify quality and purity. cDNA synthesis was performed using first strand cDNA synthesis kit (Roche, Switzerland) according to the manufacturer's instructions. Real-time PCR was performed in $20 \mu \mathrm{L}$ reactions on cDNA with SYBR Green PCR reagents (Roche) using CFX96 Touch TM Real-Time PCR Detection System (Bio-Rad, USA). The level of target mRNA was normalized to the level of GAPDH (B661104, Sangon Biotech, China) and compared with control. Data were analyzed using $2^{-\Delta \Delta C T}$ method. Each gene analysis was performed in triplicate. Primer's sequences of the targeted genes are listed in Table 1.

\section{Western blot}

The cells were lysed in RIPA (P0013B, Beyotime) with $1 \%$ PMSF (ST506, Beyotime) on ice for $5 \mathrm{~min}$ and removed with a scraper. Then, the lysate was centrifuged at $16,100 \mathrm{~g}$ for $5 \mathrm{~min}$, and the supernatant was collected. The samples were diluted with SDS-PAGE Sample Loading Buffer (Beyotime) and kept at $100{ }^{\circ} \mathrm{C}$ for $10 \mathrm{~min}$. Proteins were separated in $8 \%$ to $12 \%$ sodium dodecyl sulfate polyacrylamide gel electrophoresis (according to the molecular weights) and transferred to a PVDF membrane (FFP28, Beyotime) at $200 \mathrm{~mA}$ for $1.5 \mathrm{~h}$ at $4{ }^{\circ} \mathrm{C}$. The membrane was washed twice with Milli-Q water, stained with Ponceau $\mathrm{S}$ for protein visualization, and washed three times with $2 \% \mathrm{v} / \mathrm{v}$ TBST (tris-buffered saline with Tween-20). The blot was blocked with QuickBlock $^{\text {tw }}$ Blocking Buffer (P0228, Beyotime) for $2 \mathrm{~h}$ at RT and incubated separately with the following primary antibodies: COL-II (dilution of 1:5000), Aggrecan (dilution of 1:100), SOX9 (dilution of 1:5000), MMP-13 (dilution of 1:3000), COL-X (dilution of 1:250), Runx2 (dilution of 1:1000), COL-I (dilution of 1:5000), $\alpha$-SMA (dilution of 1:1000), p-AMPK (dilution of 1:1000, AF3423, Affinity), AMPK (dilution of 1:1000, DF6361, Affinity), p-PI3K (dilution of 1:1000, AF3242, Affinity), PI3K (dilution of 1:1000, AF6241, Affinity), p-AKT (dilution of 1:1000, AF908, Affinity), AKT (dilution of 1:1000, AF6261, Affinity), and GAPDH (dilution of 1:5000; ab8245, Abcam) for overnight at $4{ }^{\circ} \mathrm{C}$. Next, the membrane was washed four times with TBST for $10 \mathrm{~min}$, incubated with Goat Anti-Mouse IgG $(\mathrm{H}+\mathrm{L})$ (dilution, 1:

Table 1 Sequences of the primers used in this study

\begin{tabular}{lll}
\hline Name & Forward & Reverse \\
\hline COL2a1 & TGCTGCCCAGATGGCTGGAGGA & TGCCTTGAATCCTTGAGGCCC \\
ACAN & TCCTGGTGTGCTGCTGTCC & TCTGGCTCGGTGGTGAACTCTAG \\
MOX9 & GACTTCCGCGACGTGGAC 13 & GTTGGGGGCAGGTACTG \\
Runx2 & TCCTGGCTGCCTTCCTCTTCTTG & AGTCATGGAGCTTGCTGCATTCTC \\
COL10a1 & AACAGCAGCAGCAGCAGCAG & GCACCGAGCACAGGAAGTTGG \\
COL1a1 & GCCACCAGGCATTCCAGGATTC & GGAAGACCAGGCTCTCCAGAGTG \\
a-SMA & GCGAGAGCATGACCGATGGATTC & GCCTTCTTAGGTGCCAGTCTG \\
GAPDH & TCGTGCTGGACTCTGGAGATGG & CCGATGAAGGATGGCTGGAACAG \\
\hline
\end{tabular}


2000; SA00001-1; proteintech, China) or Goat AntiRabbit IgG (H+L) (dilution, 1:2000; SA00001-2; proteintech) secondary antibody for $1 \mathrm{~h}$ at RT, washed again four times, and visualized with Western ECL Substrate (Thermo Scientific) for chemiluminescence.

\section{Rat OA model}

Male Sprague-Dawley (SD) rats (10 weeks old) were purchased from Army Medical University (Chongqing, China). All animal experiments were conducted in accordance with the guidelines of the Animal Experiments Committee and approved by the Institutional Review Board of Southwest Hospital. The experimental mice were subjected to surgically induced OA by anterior cruciate ligament transection (ACLT) as previously described [20]. The animals were divided into three groups: sham $(n=10)$, ACLT $(n=10)$, and ACLT+AA $(n=10)$. Rats in the ACLT+AA group were followed by intra-articular injection with $0.1 \mathrm{~mL}$ of AA $(2.5 \mu \mathrm{g} / \mathrm{mL})$ into the articular cavity once a week for 8 weeks, while rats in the ACLT group were injected with $0.1 \mathrm{~mL}$ of vehicle $(0.9 \% \mathrm{NaCl})$ as a control. Food and water were available ad libitum. Rats were maintained under a constant temperature of $20 \pm 2{ }^{\circ} \mathrm{C}$, a relative humidity of $50 \% \pm 10 \%$, and a 12 -h light/dark cycle. At 4 and 8 weeks post-AA intra-articular injection, 5 rats for each group were sacrificed, and knee joint tissues were collected for further evaluation.

\section{Macroscopic observation and histological analysis}

Macroscopic evaluation was performed by five blinded investigators (LP, YJ, GL, FZ, and LR). The erosion of articular cartilage was graded according to the macroscopic score system [21].

Knee joint samples $(n=3)$ were fixed in $4 \% \mathrm{v} / \mathrm{v}$ paraformaldehyde for 2 days, and tissues were decalcified in 10\% wt\% EDTA disodium salt dihydrate (GRM1195, neofroxx, Germany) solution for 4 weeks at RT. After that, the samples were dehydrated through an alcohol gradient, cleared, and embedded in paraffin blocks. Frontal serial sections ( $4 \mu \mathrm{m}$ thick) across entire joints were obtained and then stained with Safranin-O/Fast Green to assess cartilage destruction. The stained sections were photographed digitally under a microscope. Histologic changes in the medial tibial plateau and medial femoral condyle of knee joints were scored on a scale of 0-6 according to the recommendations of the Osteoarthritis Research Society International (OARSI) scoring system [22].

\section{Immunohistochemical analysis}

Immunohistochemistry (IHC) was performed to evaluate the phenotype of articular chondrocytes. After deparaffinization and hydration with distilled water, the antigen repair was conducted at $37^{\circ} \mathrm{C}$ for $10 \mathrm{~min}$. Then, the tissue slices were penetrated with PBS for 5 min followed with $\mathrm{H}_{2} \mathrm{O}_{2}$ treatment for about $20 \mathrm{~min}$, and then blocked for $60 \mathrm{~min}$ to avoid the homologous serum.

Sections were incubated overnight at $4{ }^{\circ} \mathrm{C}$ with antibodies against the following proteins: COL-II (dilution of 1:200), MMP-13 (dilution of 1:100), COL-X (dilution of 1:80), Runx2 (dilution of 1:500), COL-I (dilution of 1: 200 ), and $\alpha$-SMA (dilution of 1:200). After rinsing with PBS, sections were incubated with appropriate biotinylated secondary antibody and horseradish peroxidaseconjugated streptavidin-biotin. Immunoreactivity was visualized with a 3,3'-diaminobenzidine tetrahydrochloride kit (ZSGB Bio, China) followed by counterstaining with methyl green. The presence of antigen in the cartilage was estimated by calculating the number of chondrocytes that stained positive. The total number of chondrocytes and those that stained positive in three central regions of articular cartilage were counted using Image Pro Plus version 5.1 software (Media Cybernetics, USA). The percentage of positive stained cells for the antigen and the relative fold change of different groups were then determined.

\section{Statistical analysis}

Data were expressed as mean \pm standard deviation (SD). Statistical significance was assessed by one-way analysis of variance (ANOVA) (more than two groups) or unpaired $t$ tests (two groups). $P<0.05$ was considered statistically significant.

\section{Results}

\section{Effects of AA on chondrocyte viability}

The chemical structure of AA is shown in Fig. 1a. The live-dead staining results of chondrocytes after AA treatment are shown in Fig. 1b. Chondrocytes treated with $5 \mu \mathrm{M}$ AA showed comparable staining with untreated control, while $10 \mu \mathrm{M}$ and $20 \mu \mathrm{M}$ AA treatment induced more dead chondrocytes. CCK-8 assay (Fig. 1c) was used to quantitatively evaluate the viability of AA-treated chondrocytes. Consistent with live-dead staining results, $5 \mu \mathrm{M}$ AA treatment did not affect chondrocyte viability (compared with control), while $10 \mu \mathrm{M}(P<0.01$, compared with control) and $20 \mu \mathrm{M}(P<0.0001$, compared with control) AA treatment significantly reduced chondrocyte viability. Therefore, $5 \mu \mathrm{M}$ AA was used for the subsequent experiments.

\section{Effects of AA on hypertrophy marker expression in chondrocytes}

To analyze whether AA inhibited chondrocyte hypertrophy, we measured hypertrophic markers COL-X, MMP-13, and Runx2 using immunofluorescence staining, RT-PCR, and western blot analysis. In human OA 

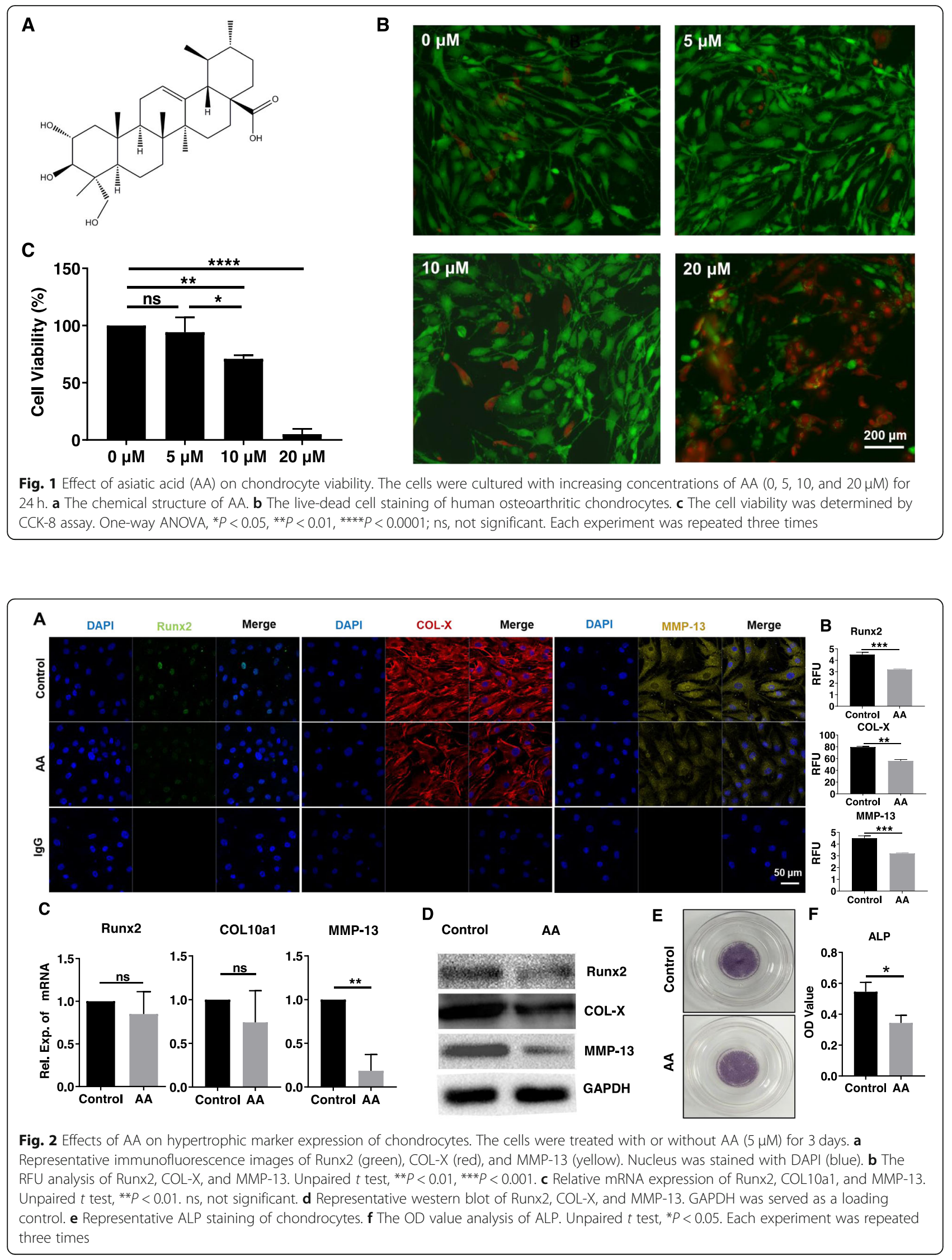
chondrocytes, AA treatment reduced expression of Runx2, COL-X, and MMP-13 protein when compared to the control group, as demonstrated by immunofluorescence staining and its RFU analysis (Fig. 2a, b). RT-PCR results showed that the mRNA level of Runx2 and COL10a1 in the AA group was lower than that in the control group, but with no statistical difference (Fig. 2c); the mRNA level of MMP-13 in the AA group was significantly lower than that in the control group $(P<0.01)$. Western blot results further revealed that (Fig. 2d) the protein levels of Runx2, MMP-13, and COL-X in the AA group were reduced in different degrees, as compared with the control group. Additionally, a weaker staining of ALP after AA treatment was observed when compared to the control group (Fig. 2e, f). The reduction in ALP further confirmed the antihypertrophic effect of AA.
Effects of AA on fibrosis factor expression in chondrocytes

To establish if AA inhibited key regulators of chondrocyte fibrosis, the expression of COL-I and $\alpha$-SMA was assessed. We found that the staining of COL-I and $\alpha$ SMA in the AA group was weaker compared with the control group (Fig. 3a, b). COL1a1 mRNA level was significantly reduced by $\sim 50 \%$ while $\alpha$-SMA mRNA was significantly reduced by $\sim 22 \%$ in the AA group compared with the control group (Fig. 3c). Consistently, protein (Fig. 3d) levels of COL-I and $\alpha$-SMA were also significantly inhibited by AA treatment.

Effects of AA on chondrocytic phenotype of chondrocytes We further analyzed the effects of AA treatment on chondrocytic phenotype. It was shown that the immunofluorescence staining and its RFU analysis results of ACAN, COL-II, and SOX9 in the AA group were

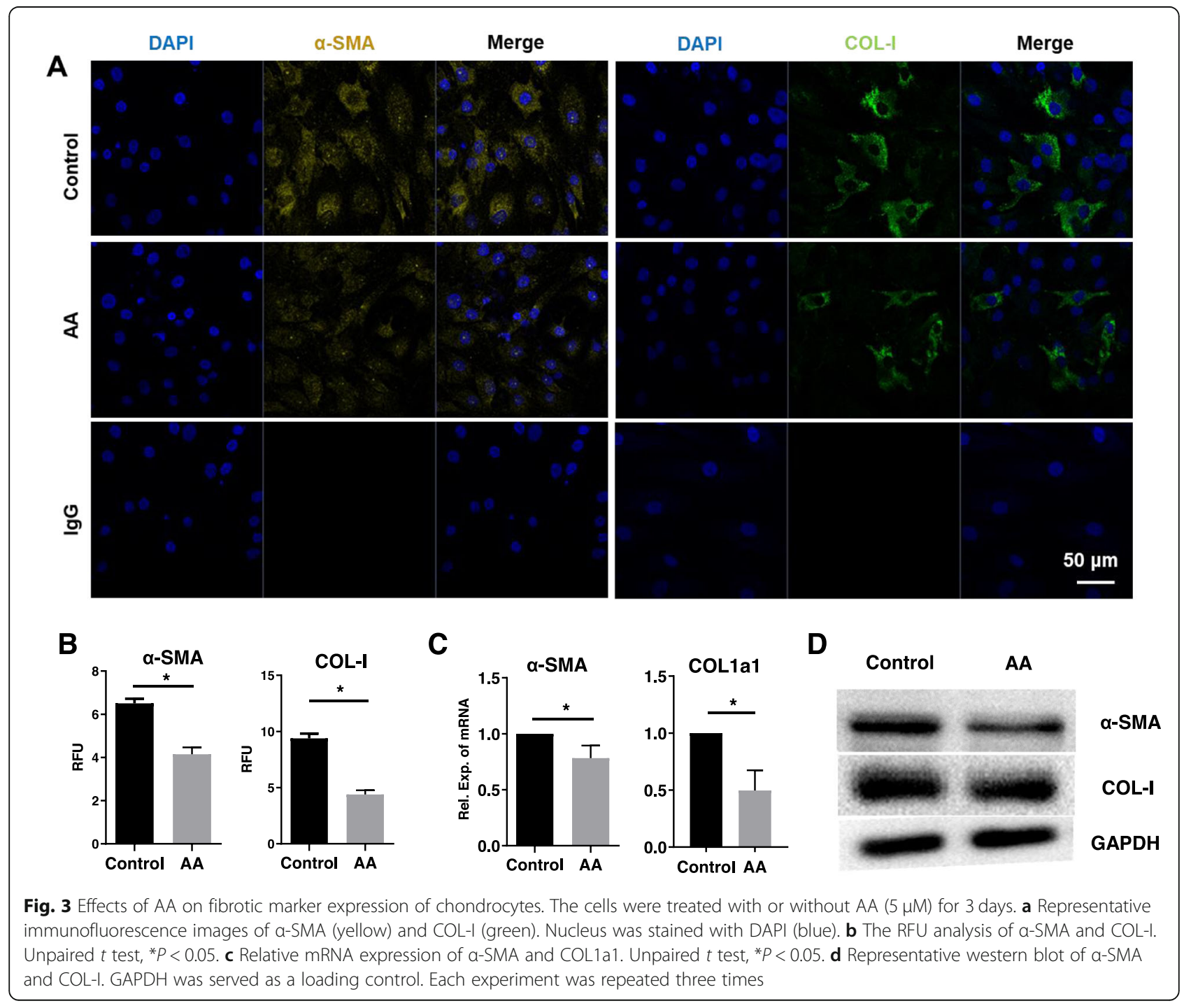


comparable with the control group (Fig. 4a, b). Although gene expression of COL2a1, ACAN, and SOX9 in the AA group was much higher than that in the control group (Fig. 4c), the protein levels of these markers were similar between the AA group and control group (Fig. 4d). In addition, the Alcian Blue staining showed no obvious difference between the control group and AA group as well (Fig. 4e).

\section{AA regulated the AMPK/PI3K/AKT signaling pathway}

To define further the molecular mechanism for the AAmediated anti-hypertrophy and anti-fibrosis of human OA chondrocytes, we evaluated the potential role of the AMPactivated protein kinase (AMPK) and phosphoinositide-3 kinase/protein kinase B (PI3K/AKT) signaling pathway. After AA treatment, we found that the phosphorylation of AMPK was increased and the phosphorylation of PI3K and AKT was significantly decreased in the AA group (Fig. 5a) compared with the control group. Meanwhile, the ratio of phosphorylated AMP-activated protein kinase (p-AMPK)/AMPK was increased by $\sim 23 \%$ and the ratios of phosphorylated phosphoinositide-3 kinase (p-PI3K)/ PI3K and phosphorylated protein kinase B (p-AKT)/AKT were decreased by $\sim 48 \%$ and $\sim 40 \%$, respectively (Fig. 5 b). These results suggest that AA protected the chondrocytes against chondrocyte hypertrophy and chondrocyte fibrosis by activating AMPK and inhibiting the PI3K/AKT signaling pathway.

\section{Effects of AA on the cartilage destruction in ACLT rat model}

The process of animal experiment is shown in Fig. 6a. Starting 1 month after the ACLT surgery, the following 4 weeks and 8 weeks were used as time points for observation. The knees in each group were isolated for macroscopic observation (Fig. 6b). Cartilage on the femoral condyles in the sham group appeared macroscopically normal with a smooth surface, and no cartilage defects or osteophytes were observed at 4 and 8 weeks. In the ACLT group, the cartilage surface was uneven and rough at 4 weeks and got eroded with local ulcers at 8 weeks. Interestingly, the cartilage surface in the ACL$\mathrm{T}+\mathrm{AA}$ group was as smooth as that in the sham group.

Histological analysis of cartilage was performed using Safranin O-Fast Green staining (Fig. 6c). We found that the cartilage surface was smooth and showed positive red staining in the sham group. Compared with the ACLT group, the ACLT+AA group showed a milder severity of OA caused by ACLT surgery (Fig. 6c). The ACLT group showed cartilage erosion, apparent hypocellularity, and massive proteoglycan loss compared with the ACLT+AA group. Moreover, histochemical analysis

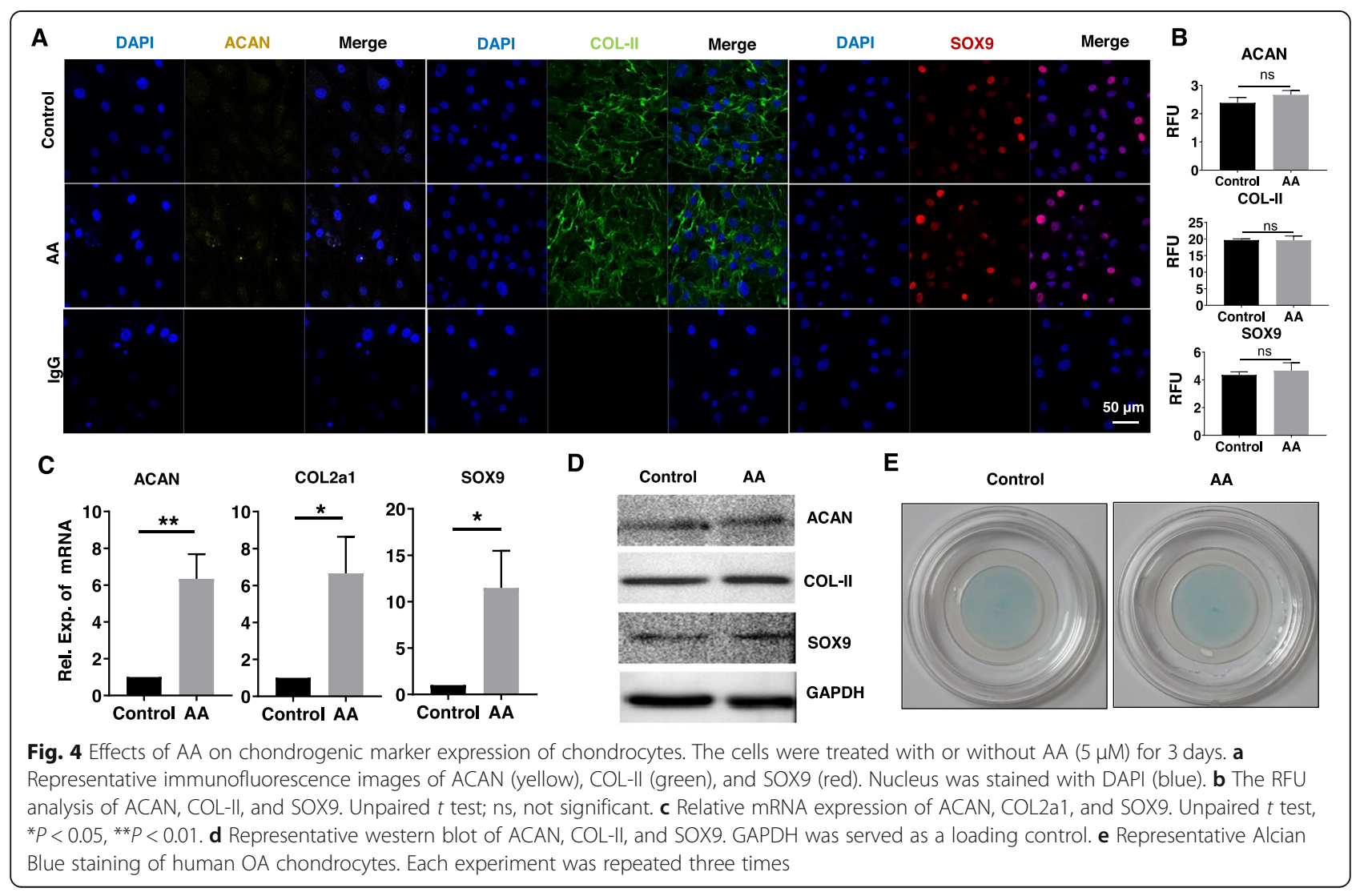




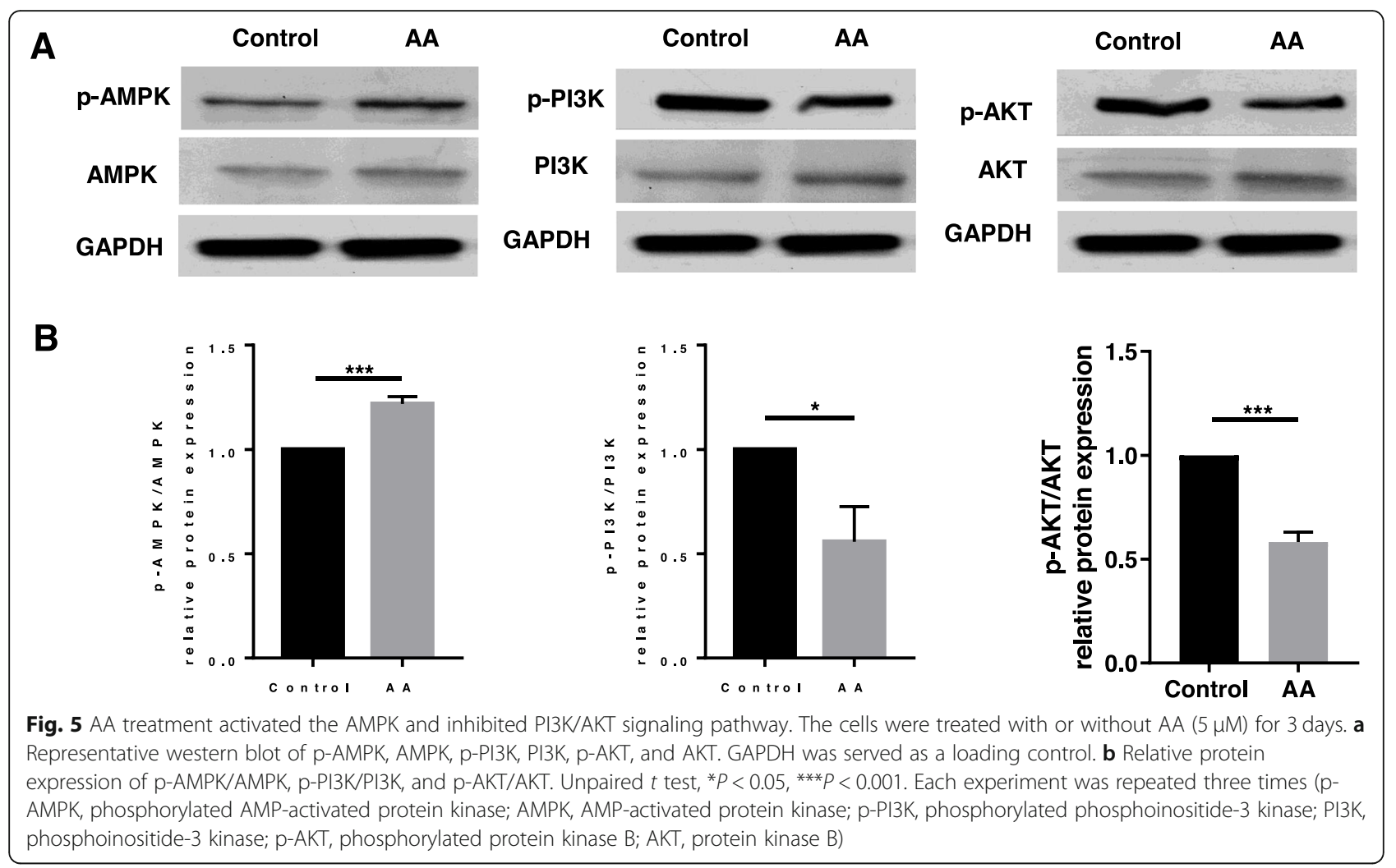

showed that COL-II expression was decreased in the ACLT group compared with the sham and ACLT+AA group (Fig. 6d). The macroscopic observation scores and the OARSI scores were utilized to quantify the severity of cartilage damage (Fig. 6e, f). All these results suggested that AA slowed down the degeneration of cartilage matrix during OA development.

\section{Effects of AA on chondrocyte hypertrophy in rat $O A$ model}

Histochemical analysis revealed that ACLT operation induced COL-X, MMP-13, and Runx2 expression in the proximal tibia of articular cartilage of rats at 4 and 8 weeks post-intra-articular injection (Fig. 7a, c). Injection of AA reduced this induction, as was shown by the limited expression of COL-X, MMP-13, and Runx2 in the ACLT+AA group. The positive cells expressing COL-X, MMP-13, and Runx2 in the ACLT+AA group were much fewer than those in the ACLT group at 4 and 8 weeks (Fig. 7d). Taken together, these results indicated that AA attenuated the hypertrophy of chondrocytes in ACLT-induced rat OA model.

\section{Effects of $A A$ on chondrocyte fibrosis in rat OA model}

To evaluate the fibrosis of chondrocytes, histochemical analysis was performed to analyze the expression of COL-I and $\alpha$-SMA in the proximal tibia of articular cartilage of rats at 4 and 8 weeks post-intra-articular injection (Fig. 8a, b). We found an elevated expression of COL-I and $\alpha$-SMA expression in rat knee joints after the ACLT operation, but AA injection partly reversed the trend. In addition, quantitative data showed that the positive cells expressing COL-I and $\alpha$-SMA in the ACL$\mathrm{T}+\mathrm{AA}$ group were fewer than those in the ACLT group at 4 and 8 weeks (Fig. $8 \mathrm{c}, \mathrm{d}$ ). Taken together, these data revealed that AA repressed the chondrocyte fibrosis during OA development.

\section{Discussion}

Articular chondrocytes tend to lose phenotypic stability when the microenvironment changes. In cartilagerelated diseases such as $\mathrm{OA}$, chondrocytes gradually acquire a hypertrophic phenotype; in in vitro culture, chondrocytes normally obtain a fibrotic phenotype. Therefore, how to avoid the unwanted phenotype and maintain the chondrogenic phenotype is a concern in chondrocyte biology. In this study, we found that AA could reduce chondrocyte hypertrophy and fibrosis via AMPK/PI3K/AKT signaling pathway.

First, we assayed the effect of AA on the human OA chondrocyte viability. Compared with $10 \mu \mathrm{M}$ and $20 \mu \mathrm{M}$ AA, $5 \mu \mathrm{M}$ AA showed comparable staining with untreated control and less dead chondrocytes, which was further verified by CCK- 8 assay. These results indicated that the range of concentration selection for future clinical use is relatively small, which needs more accurate 


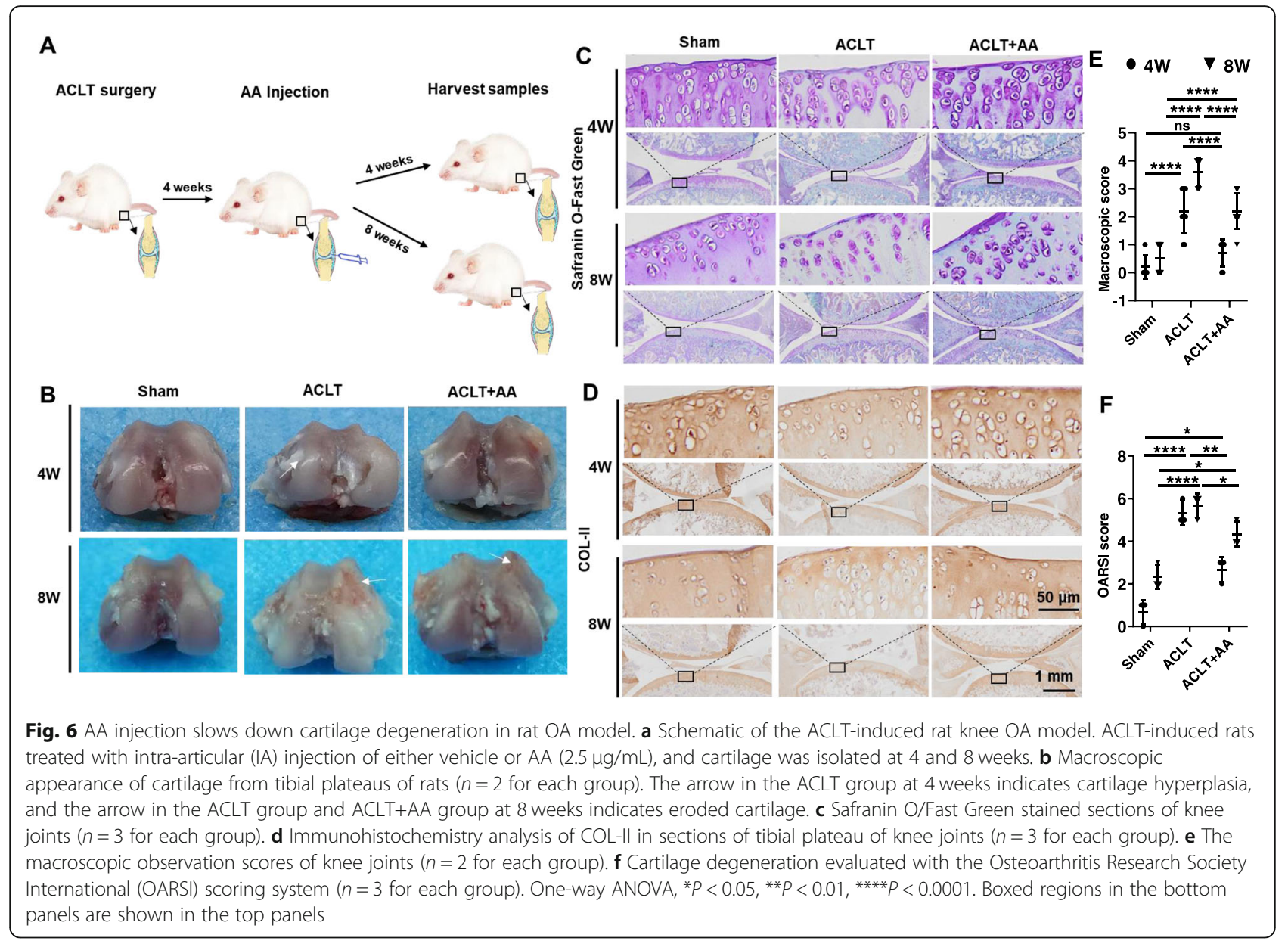

understanding of mechanism to keep the precise control of dosage.

Then, we detected the change of hypertrophic markers COL-X, MMP-13, and Runx2 in human osteoarthritic chondrocytes after $5 \mu \mathrm{M}$ AA treatment. As expected, AA downregulated the expression of COL-X, MMP-13, and Runx2 at protein level. Moreover, AA treatment induced a weaker ALP staining compared with untreated control. Similar with our results, $\mathrm{Gu}$ et al. found that resveratrol inhibited chondrocyte hypertrophy through suppressing the expression of MMP-13 in human articular chondrocytes [23]; Yahara et al. showed that Pterosin B prevented chondrocyte hypertrophy via downregulating the expression of COL-X, Runx2, and ALP [24].

In addition, we found that AA also effectively inhibited chondrocyte fibrosis. Note that in situ chondrocyte fibrosis tends to be connected with the onset of OA $[25,26]$. The fibrotic remodeling of osteoarthritic cartilage enhanced by the presence of COL-I [27] and the secretion of $\alpha$-SMA by osteoarthritic chondrocytes has been verified [28]. Our study showed that AA treatment lowered the expression of COL-I and $\alpha$-SMA both in vitro and in vivo, indicating that the fibrotic differentiation of chondrocytes was attenuated by AA.
It has been shown that changes in collagen types from COL-II to COL-I, COL-III, and COL-X within OA cartilage participate to OA physiopathology by damaging the assembly, integrity, and stability of the extracellular matrix synthesized $[29,30]$. Report on rabbit articular chondrocytes showed a final "dedifferentiated" phenotype, as $41 \%$ of COL-I, $25 \%$ of $\mathrm{X}_{2} \mathrm{Y}, 20 \%$ of COL-I trimer, $13 \%$ of COL-III, and $1 \%$ of COL-II [12]. In normal articular cartilage, COL-II is the more prominent collagen found associated in heteropolymer with COL-IX and COL-XI ( 1\% COL-IX, $\sim 3 \%$ COL-XI, $\geq 90 \%$ COLII). In accordance with these observations, authors proposed to consider COL-II/COL-I ratio as a signature for the dedifferentiation process [31]. These researches indicated that hypertrophic-like and fibroblastic-like phenotypes were increasing while chondrogenic phenotypes were decreasing during OA progression. Hence, we further studied the effects of AA treatment on the chondrogenic phenotype of chondrocytes. Chondrogenesis is characterized by the expression of SOX9, COL2a1, and ACAN. In conjunction with SOX5 and SOX6 [32] and interacting with $\mathrm{CEBP} / \mathrm{p} 300$ [33], SOX9 activates the expression of chondrocyte-specific genes such as COL2a1 


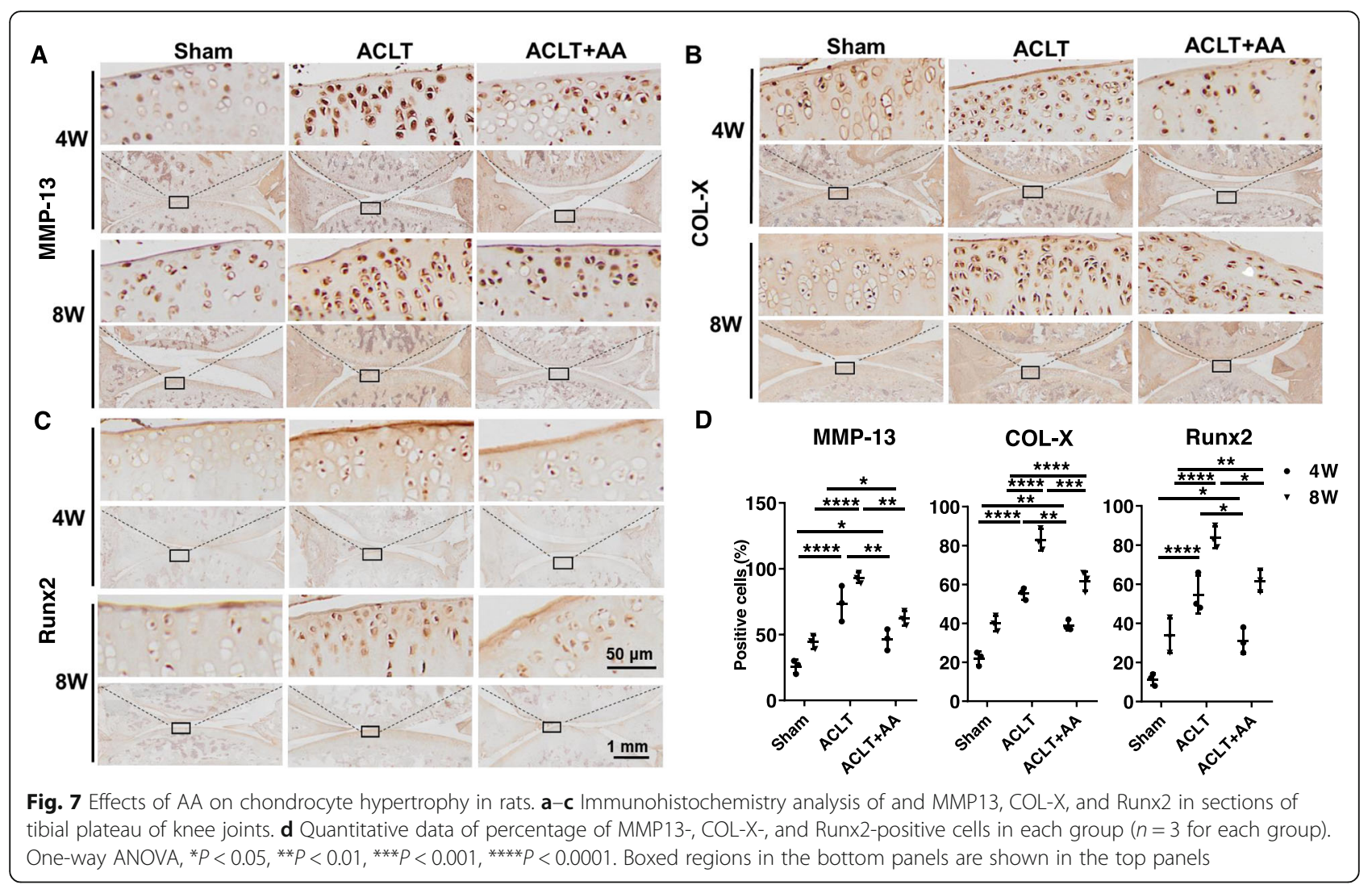

and ACAN, leading to deposition of COL-II and proteoglycans. However, we found that there was an increase expression of these chondrogenic markers (SOX9, COLII, and ACAN) in the gene level but no significant difference in the protein level between AA-treated chondrocytes and untreated control. In fact, the mRNA abundance of a particular gene does not necessarily have a linear relationship with the expression of its translation product protein, because there are many levels of regulation of gene expression. mRNA degradation, protein degradation, modified folding, and other factors may lead to the expression difference between gene and protein level.

Based on these results, we further explored the mechanism of AA on anti-hypertrophic and anti-fibrosis in vitro. Previous studies have shown that certain signaling pathways are very important in regulating chondrocyte differentiation. For example, BMP2 and BMP4 induce hypertrophy during the chondrogenic differentiation of human MSCs in vitro [34]. In the presence of TGF- $\beta$, human MSCs show a significant enhancement of chondrogenesis and suppression of hypertrophic differentiation [35]. Moreover, postnatal WNT/ $\beta$-catenin signaling in growth plate promotes hypertrophic differentiation and endochondral ossification [36]. Reinhold et al. indicated that canonical WNT signaling induces the transcription repressor Twist1, which strongly inhibits chondrocyte gene expression [37]. However, the role of PI3K/AKT pathway remains puzzling: some studies have reported a positive [38] while others a negative [39] effect on chondrocyte hypertrophy. In this study, we found that AA treatment attenuated chondrocyte hypertrophy and fibrosis through downregulating the phosphorylation of PI3K/AKT signaling pathway. In contrast, after activation of PI3K at the leading edge, AKT rapidly accumulates by binding to PtdIns $(3,4,5) \mathrm{P}_{3}$ via its pleckstrin homology domain, leading to activation of AKT by phosphorylation. The PI3K/AKT pathway was shown to be involved in Runx2-dependent osteoblast and enhanced DNA binding of Runx2 and Runx2-dependent transcription [38]. A previous study suggested that AKT enhances Runx2 protein stability by regulating Smurf2 function during osteoblast differentiation [40]. Another study also found that the PI3K/AKT pathway was required for hypertrophic differentiation. The major phenotype of the LY294002-treated tibiae was represented by a $45 \%$ reduction in the length of the hypertrophic zone [41]. In addition, AKT1 and AKT2, but not AKT3, were reported to inhibit fibrogenesis in hepatocytes and HSC [42]. Besides, previous studies have revealed that the PI3K/AKT signaling pathway is associated with hypertrophy and fibrosis in different tissues such as cardiac hypertrophy [43] and liver fibrosis [18]. Moreover, our data also found that AA treatment 


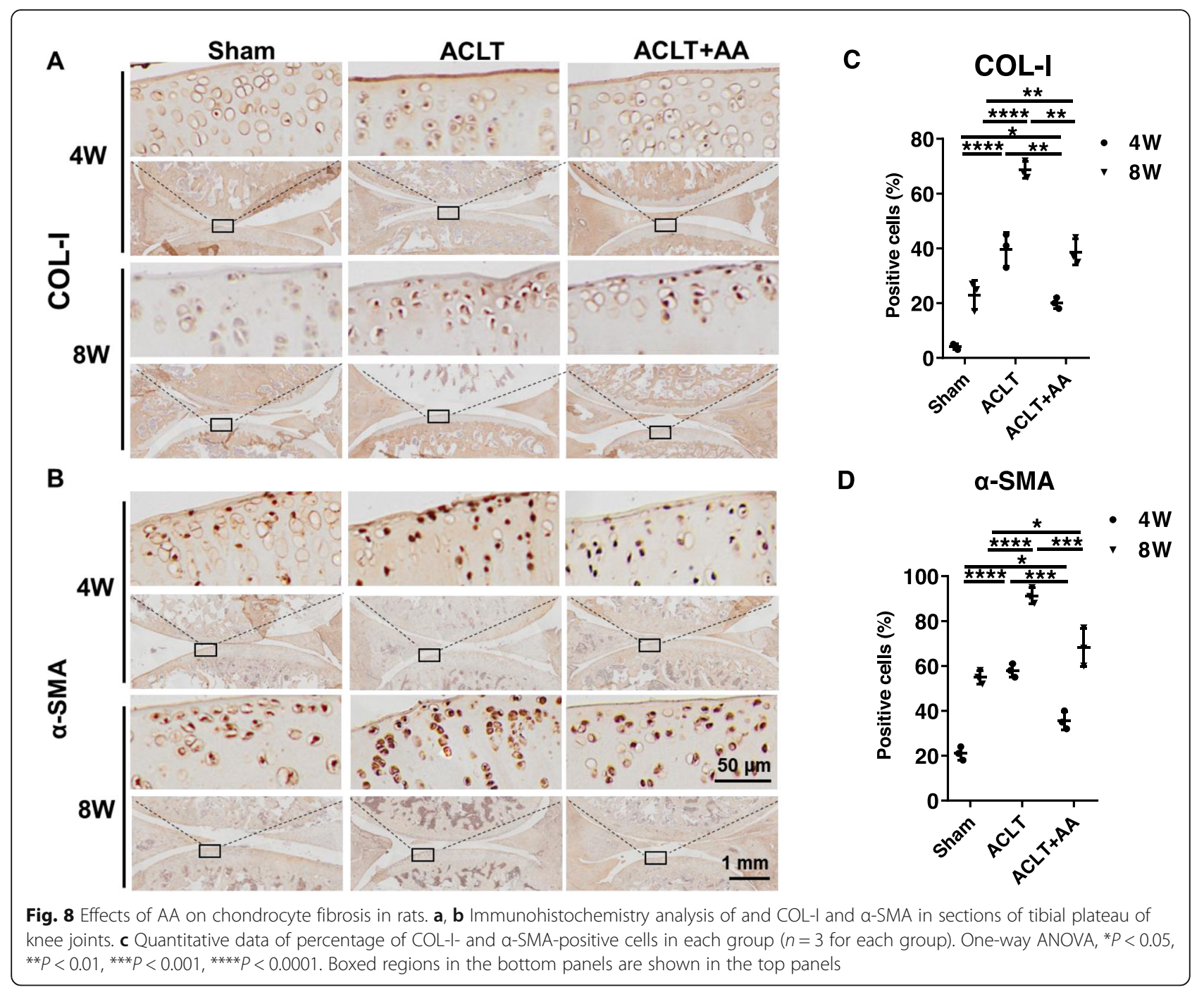

upregulated the phosphorylation of AMPK. A previous study has shown that the activation of AMPK could inhibit PI3K/AKT signaling via inhibition of PtdIns $(3,4,5)$ $\mathrm{P}_{3}$ accumulation at the plasma membrane [44]. Hence, these results have indicated that AA might attenuate chondrocyte hypertrophy and fibrosis via AMPKmediated PI3K/AKT signaling inhibition.

Interestingly, it is reported that loss of AMPK signaling changes downstream signaling and induces proinflammatory cytokines that potentially cause insulin resistance [45]. Another study shows that chondrocyte differentiation is functionally associated with decreased AMPK activity. Treatment with metformin, an activator of AMPK, significantly reduced cartilage matrix formation and inhibited gene expression of SOX6, SOX9, COL2a1, and aggrecan core protein (acp) [46]. However, our data showed no significant changes of chondrocytic phenotypes between the control group and AA-treated group, which indicated that AA might regulate the expression of chondrocytic phenotypes and keep SOX9 in balance via some other pathways. Future work is expected to explore the exact mechanism of AA on regulating chondrocyte differentiation. Beyond that, the study has shown that SOX9 can both interact directly with and suppress Runx 2 activity and other genes related to hypertrophic chondrocytes, such as Col10a1 [47-49]. These researches implicated that AA might also alleviate hypertrophic differentiation via keeping the expression of SOX9 in balance.

In our vivo experiment, the results showed that AA had a chondroprotective effect on ACLT rat model. Meanwhile, AA significantly reduced the expression of hypertrophic and fibroblastic phenotypes in both 4 and 8 weeks, which indicated that AA showed a curative effect on alleviating the OA progression. Taken together, these results reveal the modulation of AMPK/PI3K/AKT plays an essential part in the regulation of hypertrophy and fibrosis in human OA chondrocytes treated with 


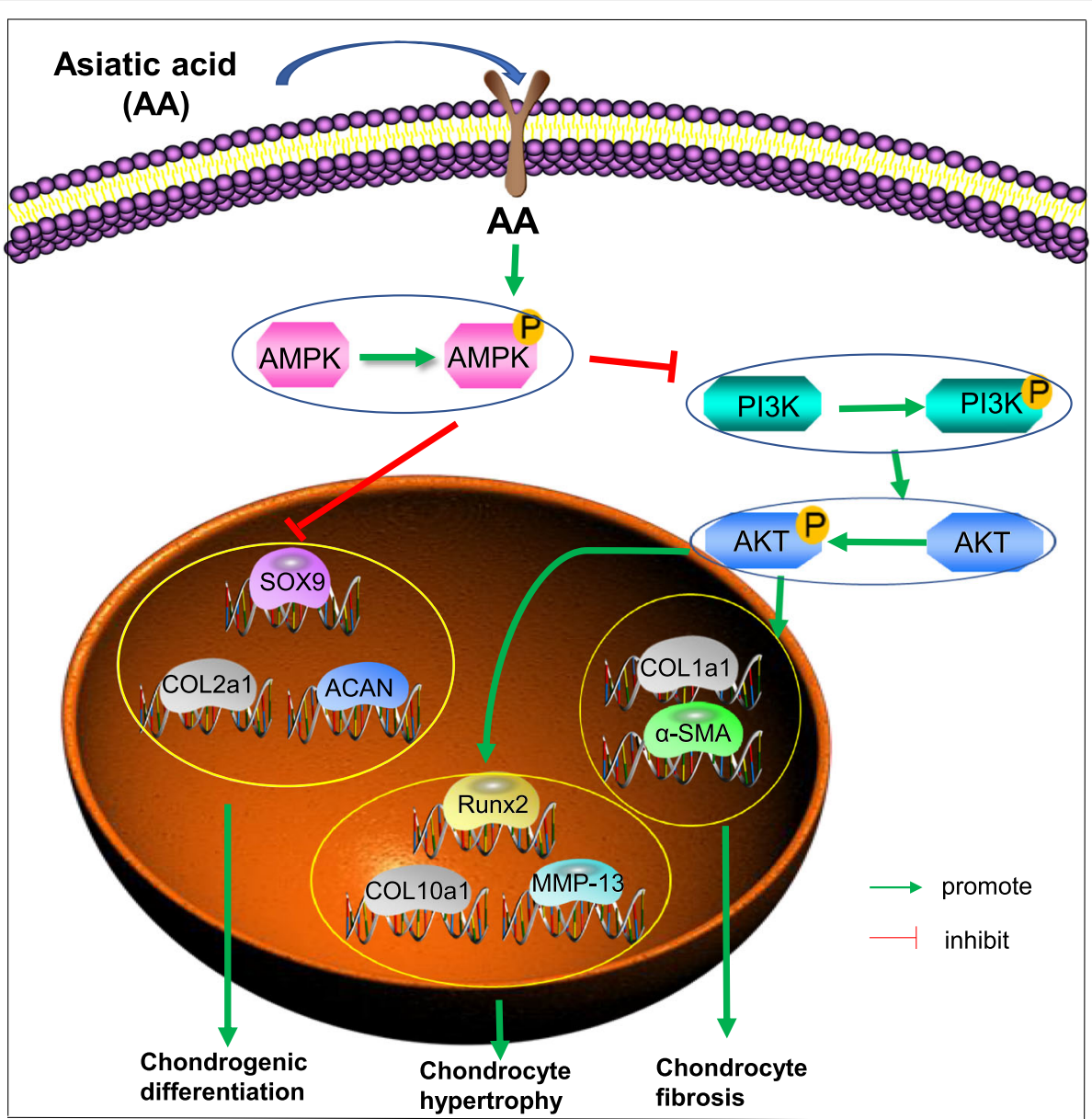

Fig. 9 Schematic diagram of the mechanism of AA on attenuating hypertrophic and fibrotic differentiation

AA in vitro (Fig. 9). It is worthy to note that ACLT surgery + AA injection was performed on relatively young rats in our study. In this case, the response of rat chondrocytes might not fully reflect the response of human osteoarthritic chondrocytes from aged patients to AA injection.

\section{Conclusions}

In conclusion, AA treatment could reduce hypertrophic and fibrotic differentiation and maintain the chondrogenic phenotype of articular chondrocytes mainly via AMPK/PI3K/AKT signaling pathway. Our study suggested that AA might be a prospective drug component that targets hypertrophic and fibrotic chondrocytes for OA treatment.

\section{Abbreviations}

OA: Osteoarthritis; AA: Asiatic acid; COL-X: Type X collagen; MMP-13: Matrix metalloproteinase-13; ALP: Alkaline phosphatase; Runx2: Runt-related transcription factor 2; COL-I: Type I collagen; a-SMA: Alpha-smooth muscle actin; SOX9: SRY-related HMG box 9; COL-II: Type II collagen;

ACAN: Aggrecan; ACLT: Anterior cruciate ligament transection; $p$ AMPK: Phosphorylated AMP-activated protein kinase; AMPK: AMP-activated protein kinase; p-PI3K: Phosphorylated phosphoinositide-3 kinase;
PI3K: Phosphoinositide-3 kinase; p-AKT: Phosphorylated protein kinase B; AKT: Protein kinase B; DMSO: Dimethylsulfoxide; OARSI: Osteoarthritis Research Society International; RT-PCR: Reverse transcription-polymerase chain reaction; SD: Standard deviation; ANOVA: Analysis of variance; RFU: Relative fluorescence unit

\section{Acknowledgements}

The authors would also like to acknowledge Yang Qi for his technical expertise with painting the chemical structure of $A A$.

\section{Authors' contributions}

LN, CC, and YL conceived and designed the study. LN, LPJ, YJJ, FDJ, CJJ, LR and WX performed the experiment. LN, FZL, SXB, GXY, CC, and YL analyzed and interpreted the data. LN wrote the paper. All authors have read and approved the final manuscript.

\section{Funding}

This work was supported by the National Natural Science Foundation of China (grant nos. 81572107 and 31800784).

Availability of data and materials

The datasets analyzed during the current study are available from the corresponding author on reasonable request.

Ethics approval and consent to participate

Cartilage samples were obtained intraoperatively from patients undergoing total knee arthroplasties with approval from the Ethics Committee of Southwest Hospital. All animal experiments were authorized by the Animal 
Care and Use Committee of Third Military Medical University (Army Medical University).

\section{Consent for publication}

Not applicable.

\section{Competing interests}

The data presented in this manuscript are original and have not been published or submitted elsewhere. All listed authors have approved the manuscript and agreed with the submission. The authors declare that they have no conflict of interest.

\section{Author details}

${ }^{1}$ Key Laboratory of Freshwater Fish Reproduction and Development, Ministry of Education, Laboratory of Molecular Developmental Biology, School of Life Sciences, Southwest University, Chongqing, China. ${ }^{2}$ Center for Joint Surgery, Southwest Hospital, Third Military Medical University (Army Medical University), Chongqing, China. ${ }^{3}$ Zunyi Traditional Chinese Medicine Hospital, Zunyi, China. ${ }^{4}$ Biomedical Analysis Center, Third Military Medical University (Army Medical University), Chongqing, China. ${ }^{5}$ College of Medical Informatics, Chongqing Medical University, Chongqing, China.

\section{Received: 4 December 2019 Accepted: 22 April 2020}

Published online: 12 May 2020

\section{References}

1. Sun MM, Beier F. Chondrocyte hypertrophy in skeletal development, growth, and disease. Birth Defects Res C Embryo Today. 2014;102(1):74-82.

2. Singh P, Marcu KB, Goldring MB, Otero M. Phenotypic instability of chondrocytes in osteoarthritis: on a path to hypertrophy. Ann N Y Acad Sci. 2019;1442(1):17-34.

3. van der Kraan PM, van den Berg WB. Chondrocyte hypertrophy and osteoarthritis: role in initiation and progression of cartilage degeneration? Osteoarthr Cartil. 2012;20(3):223-32.

4. Dreier R. Hypertrophic differentiation of chondrocytes in osteoarthritis: the developmental aspect of degenerative joint disorders. Arthritis Res Ther. 2010;12(5):216

5. von der Mark K, Kirsch T, Nerlich A, Kuss A, Weseloh G, Gluckert K, Stoss H. Type $X$ collagen synthesis in human osteoarthritic cartilage. Indication of chondrocyte hypertrophy. Arthritis Rheum. 1992;35(7):806-11.

6. Li H, Wang D, Yuan Y, Min J. New insights on the MMP-13 regulatory network in the pathogenesis of early osteoarthritis. Arthritis Res Ther. 2017 19(1):248.

7. Kudo T, Nakatani S, Kakizaki M, Arai A, Ishida K, Wada M, Kobata K Supplemented chondroitin sulfate and hyaluronic acid suppress mineralization of the chondrogenic cell line, ATDC5, via direct inhibition of alkaline phosphatase. Biol Pharm Bull. 2017:40(12):2075-80.

8. Liao L, Zhang S, Gu J, Takarada T, Yoneda Y, Huang J, Zhao L, Oh CD, Li J, Wang B, et al. Deletion of Runx2 in articular chondrocytes decelerates the progression of DMM-induced osteoarthritis in adult mice. Sci Rep. 2017;7(1): 2371.

9. Kamekura S, Kawasaki Y, Hoshi K, Shimoaka T, Chikuda H, Maruyama Z, Komori T, Sato S, Takeda S, Karsenty G, et al. Contribution of runt-related transcription factor 2 to the pathogenesis of osteoarthritis in mice after induction of knee joint instability. Arthritis Rheum. 2006;54(8):2462-70.

10. Sliogeryte K, Botto L, Lee DA, Knight MM. Chondrocyte dedifferentiation increases cell stiffness by strengthening membrane-actin adhesion. Osteoarthr Cartil. 2016:24(5):912-20.

11. Benya PD, Shaffer JD. Dedifferentiated chondrocytes reexpress the differentiated collagen phenotype when cultured in agarose gels. Cell. 1982; 30(1):215-24.

12. Benya PD, Padilla SR, Nimni ME. Independent regulation of collagen types by chondrocytes during the loss of differentiated function in culture. Cell. 1978;15(4):1313-21.

13. Lv J, Sharma A, Zhang T, Wu Y, Ding X. Pharmacological review on asiatic acid and its derivatives: a potential compound. SLAS technology. 2018;23(2): $111-27$

14. Lv H, Qi Z, Wang S, Feng H, Deng X, Ci X. Asiatic acid exhibits antiinflammatory and antioxidant activities against lipopolysaccharide and dgalactosamine-induced fulminant hepatic failure. Front Immunol. 2017;8:785.
15. Huang SS, Chiu CS, Chen HJ, Hou WC, Sheu MJ, Lin YC, Shie PH, Huang GJ. Antinociceptive activities and the mechanisms of anti-inflammation of asiatic acid in mice. Evid Based Complement Alternat Med. 201 1;2011:895857.

16. Gao J, Chen J, Tang X, Pan L, Fang F, Xu L, Zhao X, Xu Q. Mechanism underlying mitochondrial protection of asiatic acid against hepatotoxicity in mice. J Pharm Pharmacol. 2006;58(2):227-33.

17. Ma ZG, Dai J, Wei WY, Zhang WB, Xu SC, Liao HH, Yang Z, Tang QZ. Asiatic acid protects against cardiac hypertrophy through activating AMPKalpha signalling pathway. Int J Biol Sci. 2016;12(7):861-71.

18. Wei L, Chen Q, Guo A, Fan J, Wang R, Zhang H. Asiatic acid attenuates $\mathrm{CCl}$-induced liver fibrosis in rats by regulating the PI3K/AKT/mTOR and BCl2/Bax signaling pathways. Int Immunopharmacol. 2018;60:1-8.

19. Huang S, Song X, Li T, Xiao J, Chen Y, Gong X, Zeng W, Yang L, Chen C. Pellet coculture of osteoarthritic chondrocytes and infrapatellar fat padderived mesenchymal stem cells with chitosan/hyaluronic acid nanoparticles promotes chondrogenic differentiation. Stem Cell Res Ther. 2017;8(1):264

20. Lorenz J, Grassel S. Experimental osteoarthritis models in mice. Methods Mol Biol (Clifton, NJ). 2014;1194:401-19.

21. Pauli C, Grogan SP, Patil S, Otsuki S, Hasegawa A, Koziol J, Lotz MK, D'Lima DD. Macroscopic and histopathologic analysis of human knee menisci in aging and osteoarthritis. Osteoarthr Cartil. 2011;19(9):1132-41.

22. Kraus VB, Huebner JL, DeGroot J, Bendele A. The OARSI histopathology initiative - recommendations for histological assessments of osteoarthritis in the guinea pig. Osteoarthr Cartil. 2010;18(Suppl 3):S35-52.

23. Gu H, Jiao Y, Yu X, Li X, Wang W, Ding L, Liu L. Resveratrol inhibits the IL1 beta-induced expression of MMP-13 and IL-6 in human articular chondrocytes via TLR4/MyD88-dependent and -independent signaling cascades. Int J Mol Med. 2017;39(3):734-40.

24. Yahara Y, Takemori H, Okada M, Kosai A, Yamashita A, Kobayashi T, Fujita K, Itoh $Y$, Nakamura M, Fuchino $H$, et al. Pterosin B prevents chondrocyte hypertrophy and osteoarthritis in mice by inhibiting Sik3. Nat Commun. 2016;7:10959.

25. Adam M, Deyl Z. Altered expression of collagen phenotype in osteoarthrosis. Clin Chim Acta. 1983;133(1):25-32.

26. Charlier E, Deroyer C, Ciregia F, Malaise O, Neuville S, Plener Z, Malaise M, de Seny D. Chondrocyte dedifferentiation and osteoarthritis (OA). Biochem Pharmacol. 2019;165:49-65.

27. Gay S, Muller PK, Lemmen C, Remberger K, Matzen K, Kuhn K. Immunohistological study on collagen in cartilage-bone metamorphosis and degenerative osteoarthrosis. Klin Wochenschr. 1976;54(20):969-76.

28. Deroyer C, Charlier E, Neuville S, Malaise O, Gillet P, Kurth W, Chariot A, Malaise M, de Seny D. CEMIP (KIAA1199) induces a fibrosis-like process in osteoarthritic chondrocytes. Cell Death Dis. 2019;10(2):103.

29. von der Mark K, Gauss V, von der Mark H, Müller P. Relationship between cell shape and type of collagen synthesised as chondrocytes lose their cartilage phenotype in culture. Nature. 1977;267(5611):531-2.

30. Gay S, Müller PK, Lemmen C, Remberger K, Matzen K, Kühn K. Immunhistologische Untersuchungen an Kollagen bei der KnorpelKnochenumwandlung und in der degenerativen Osteoarthrose; 1976.

31. Marlovits S, Hombauer M, Truppe M, Vècsei V, Schlegel W. Changes in the ratio of type-l and type-II collagen expression during monolayer culture of human chondrocytes. J Bone Joint Surg. 2004:86(2):286-95.

32. Lefebvre $V$, Dvir-Ginzberg M. SOX9 and the many facets of its regulation in the chondrocyte lineage. Connect Tissue Res. 2016;58(1):2-14.

33. Masanao T, Shigeru T, Yuji T, Hiroshi A. Transcriptional co-activators CREBbinding protein and p300 regulate chondrocyte-specific gene expression via association with Sox9. J Biol Chem. 2003;278(29):27224-9.

34. Steinert AF, Proffen B, Kunz M. Hypertrophy is induced during thein vitrochondrogenic differentiation of human mesenchymal stem cells by bone morphogenetic protein-2 and bone morphogenetic protein-4 gene transfer. Arthritis Res Ther. 2009;11(5):R148.

35. Bian L, Zhai DY, Zhang EC, Mauck RL, Burdick JA. Dynamic compressive loading enhances cartilage matrix synthesis and distribution and suppresses hypertrophy in hMSC-laden hyaluronic acid hydrogels. Tissue Eng Part A. 2012:18(7-8):715-24

36. Akiyama H, Lyons JP, Moriakiyama Y, Yang X, Ren Z, Zhang Z, Jian MD, Taketo MM, Nakamura T, Behringer RR. Interactions between Sox9 and $\beta$ catenin control chondrocyte differentiation. Genes Dev. 2004;18(9):1072.

37. Reinhold MI, Kapadia RM, Liao Z, Naski MC. The Wnt-inducible transcription factor twist1 inhibits chondrogenesis. J Biol Chem. 2006;281(3):1381-8. 
38. Fujita T, Azuma Y, Fukuyama R, Hattori Y, Yoshida C, Koida M, Ogita K, Komori T. Runx2 induces osteoblast and chondrocyte differentiation and enhances their migration by coupling with PI3K-Akt signaling. J Cell Biol. 2004;166(1):85-95.

39. Kita K, Kimura T, Nakamura N, Yoshikawa H, Nakano T. PI3K/Akt signaling as a key regulatory pathway for chondrocyte terminal differentiation. Genes Cells. 2008;13(8):839-50.

40. Choi YH, Kim YJ, Jeong HM, Jin YH, Yeo CY, Lee KY. Akt enhances Runx2 protein stability by regulating Smurf2 function during osteoblast differentiation. FEBS J. 2014;281(16):3656-66.

41. Ulici V, Hoenselaar KD, Gillespie JR, Beier F. The PI3K pathway regulates endochondral bone growth through control of hypertrophic chondrocyte differentiation. BMC Dev Biol. 2008;8(1):40.

42. Reyes-Gordillo K, Shah R. Akt1 and Akt2 isoforms play distinct roles in regulating the development of inflammation and fibrosis associated with alcoholic liver disease. Cells. 2019;8(11):1337.

43. Li R, Shan Y, Gao L, Wang X, Wang X, Wang F. The Glp-1 analog liraglutide protects against angiotensin II and pressure overload-induced cardiac hypertrophy via PI3K/Akt1 and AMPKa signaling. Front Pharmacol. 2019;10: 537.

44. Amable G, Martinez-Leon E, Picco ME, Di Siervi N, Davio C, Rozengurt E, Rey O. Metformin inhibits beta-catenin phosphorylation on Ser-552 through an AMPK/PI3K/Akt pathway in colorectal cancer cells. Int J Biochem Cell Biol. 2019;112:88-94

45. Jeyabalan J, Shah M, Viollet B, Chenu C. AMP-activated protein kinase pathway and bone metabolism. J Endocrinol. 2012;212(3):277-90.

46. Bandow K, Kusuyama J, Kakimoto K, Ohnishi T, Matsuguchi T. AMP-activated protein kinase (AMPK) activity negatively regulates chondrogenic differentiation. Bone. 2015;74:125-33.

47. Zhou G, Zheng Q, Engin F, Munivez E, Chen Y, Sebald E, Krakow D, Lee B. Dominance of SOX9 function over RUNX2 during skeletogenesis. Proc Natl Acad Sci U S A. 2006;103(50):19004-9.

48. Hattori T, Muller C, Gebhard S, Bauer E, Pausch F, Schlund B, Bosl MR, Hess A, Surmann-Schmitt C, von der Mark H, et al. SOX9 is a major negative regulator of cartilage vascularization, bone marrow formation and endochondral ossification. Dev (Cambridge, England). 2010;137(6):901-11.

49. Zhao C, Miao Y, Cao Z, Shi J, Li J, Kang F, Dou C, Xie Z, Xiang Q, Dong S. MicroRNA-29b regulates hypertrophy of murine mesenchymal stem cells induced toward chondrogenesis; 2019.

\section{Publisher's Note}

Springer Nature remains neutral with regard to jurisdictional claims in published maps and institutional affiliations.

Ready to submit your research? Choose BMC and benefit from:

- fast, convenient online submission

- thorough peer review by experienced researchers in your field

- rapid publication on acceptance

- support for research data, including large and complex data types

- gold Open Access which fosters wider collaboration and increased citations

- maximum visibility for your research: over $100 \mathrm{M}$ website views per year

At BMC, research is always in progress.

Learn more biomedcentral.com/submissions 